\title{
Plasma parameters and electron energy distribution functions in a magnetically focused plasma
}

\author{
C. M. Samuell, ${ }^{\text {a) }}$ B. D. Blackwell, J. Howard, and C. S. Corr \\ Plasma Research Laboratory, Research School of Physics and Engineering, Australian National University, \\ Canberra, Australia
}

(Received 9 January 2013; accepted 21 February 2013; published online 12 March 2013)

Spatially resolved measurements of ion density, electron temperature, floating potential, and the electron energy distribution function (EEDF) are presented for a magnetically focused plasma. The measurements identify a central plasma column displaying Maxwellian EEDFs at an electron temperature of about $5 \mathrm{eV}$ indicating the presence of a significant fraction of electrons in the inelastic energy range (energies above $15 \mathrm{eV}$ ). It is observed that the EEDF remains Maxwellian along the axis of the discharge with an increase in density, at constant electron temperature, observed in the region of highest magnetic field strength. Both electron density and temperature decrease at the plasma radial edge. Electron temperature isotherms measured in the downstream region are found to coincide with the magnetic field lines. (C) 2013 American Institute of Physics. [http://dx.doi.org/10.1063/1.4794841]

The applied magnetic field has an important role in a number of plasma application areas including material modification, ${ }^{1}$ ion propulsion, ${ }^{2,3}$ space plasmas, ${ }^{4}$ and neutral beam injection systems for fusion. ${ }^{5}$ There are a range of magnetic field configurations that have been used on linear plasma devices, the configuration depending on the application. Recent propulsion applications have employed a magnetically diverging or converging field to induce a directed ion flow. ${ }^{3,6}$ In negative ion sources for fusion or propulsion applications, ${ }^{2,5}$ the magnetic field can be applied transverse to the discharge to achieve electron cooling and promote negative ion production.

In some applications, such as with helicon systems, the magnetic field in the plasma discharge provides an opportunity to reach high densities and very high fractional ionization. ${ }^{7,8} \mathrm{In}$ a helicon plasma, the magnetic field is applied along the axis of the system and an increase in the plasma density can be observed when a cusp or nonuniform magnetic field is located in the vicinity of the radio frequency (RF) antenna. ${ }^{9-15}$ It has also been observed that, when operated with a nonuniform magnetic field, a peaked plasma density can be obtained downstream of the production region. ${ }^{13}$ In the present work, we employ the magnetic hill (or mirror) configuration to create a high plasma density downstream of the source production region. ${ }^{16}$ This linear device is used to study materials and their interactions with plasma under fusion relevant conditions. It is important to investigate the electron transport and kinetics in these magnetized discharges. Such an investigation can be undertaken with a carefully constructed Langmuir probe, which can measure plasma parameters and the electron energy distribution function (EEDF) with very good spatial resolution. This information can then be correlated with the magnetic field topology.

The EEDF can provide valuable insight into the discharge kinetics and is therefore one of the most important plasma parameters. ${ }^{17}$ However, due to the difficult measuring environment of radio-frequency magnetized discharges, the EEDF remains poorly understood. Recently, there have been some reports of

\footnotetext{
a)cameron.samuell@anu.edu.au.
}

EEDF measurements in different types of magnetic field configurations than those described above. Aanesland et al. ${ }^{18}$ measured EEDFs and plasma parameters using a Langmuir probe across a magnetic filter in an inductively coupled plasma. It was shown that the EEDF was Maxwellian over the elastic energy range, with the electron temperature decreasing along the positive gradient of the magnetic field. In other work, Takahashi et al. ${ }^{19-21}$ studied the transport of energetic electrons across a double layer in a magnetically expanding plasma and measured a bi-Maxwellian distribution with a hot tail at the source periphery. In work by Cohen et al., ${ }^{22}$ electron populations were investigated in the Magnetic Nozzle Experiment (MNX). In that work, although the EEDF was not measured, the authors attributed the non-flat ion saturation current of the Langmuir probe current-voltage (I-V) characteristic to the formation of a suprathermal electron tail. This was supported by measurements of highly negative floating potentials, which became less negative with increasing magnetic field strength in the nozzle.

This brief communication investigates a magnetically focused plasma where the magnetic field is applied along the discharge axis, peaking in a downstream region to increase the density in that area. With this configuration, a high magnetic mirror ratio was created $\sim 45 \mathrm{~cm}$ downstream of the source production region. EEDFs are investigated alongside spatially resolved plasma parameters in such a discharge.

The measurements were performed in the recently constructed MAGnetized Plasma Interaction Experiment (MAGPIE), a magnetized linear plasma system developed at the Australian National University for plasma-material interaction studies and diagnostic development. ${ }^{16}$ The MAGPIE reactor consists of a $1 \mathrm{~m}$ long, $9.8 \mathrm{~cm}$ inner-diameter borosilicate source region connected to a $68 \mathrm{~cm}$ long stainless steel target chamber. A schematic of the MAGPIE reactor is shown in Figure 1 and a detailed description is given in Ref. 16. The plasma is created in the source region by supplying $13.56 \mathrm{MHz}$ power to an $18 \mathrm{~cm}$ long $m=1$ Nagoya III helicon antenna located approximately $2 \mathrm{~cm}$ from the source/ target interface. 


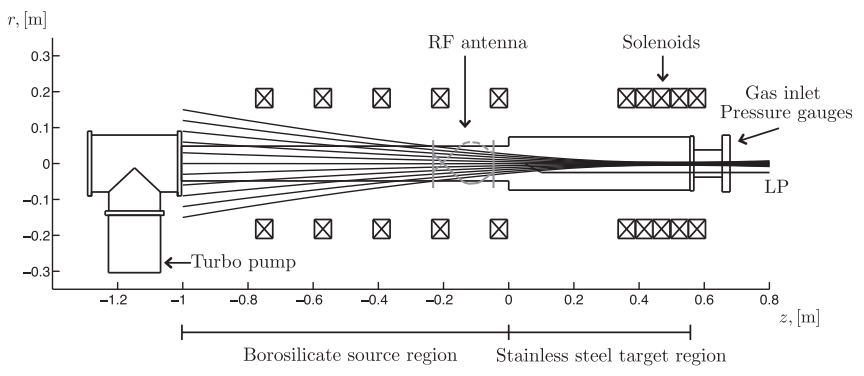

FIG. 1. Schematic of the MAGPIE reactor. The magnetic field lines given here are for an experimental setup where only the five right-most coils are active as was the case in the conditions described below.

The magnetic field is produced by two sets of watercooled solenoids that are spaced in such a way as to produce a magnetic pinch in the target region. Except where otherwise specified, the measurements described below were obtained with a $200 \mathrm{~W}, 13.56 \mathrm{MHz}$ argon plasma maintained at 1.4 mTorr. The magnetic field was produced by supplying $304 \mathrm{~A}$ to the target coils, which produced a magnetic field strength that rises from 10 to $30 \mathrm{G}$ in the antenna region to $40 \mathrm{G}$ at $z=0 \mathrm{~m}$ and about $600 \mathrm{G}$ at the pinch-point located at $z=0.45 \mathrm{~m}$. Magnetic field lines are shown in Figure 1 for this magnetic configuration. These magnetic field lines are inferred from the magnetic field produced by the superposition of a number of individual current loops. This calculation was confirmed by gauss meter measurements made at a number of locations on-axis in both the source and target chambers. ${ }^{16}$

Langmuir probe measurements were made with a custom-built rf-compensated dogleg probe along the revolution axis (z-axis) of the discharge with the probe tip orientated perpendicular to the DC magnetic field. The probe was offset from the centre of the axis by $3.1 \mathrm{~cm}$ with a fixed dogleg angle chosen so that the probe-tip passed through the radial centre of the chamber. The Langmuir probe entered through a metallic end plate and could be rotated or moved along the axis for spatially resolved measurements. The probe-tip was made from $100 \mu \mathrm{m}$ diameter tungsten wire with $6 \mathrm{~mm}$ extending beyond the surrounding pyrex probe tip holder of diameter $0.5 \mathrm{~mm}$. RF compensation was achieved with a series of tuned RF chokes positioned close to the probe tip in a method similar to that outlined by Sudit and Chen. ${ }^{23} \mathrm{~A}$ protective layer of thin copper sheet connected to the grounded probe shaft and encased in a thin glass sheath was used to shield the circuitry of the probe from stray fields and contamination. This probe design reduced the rf fluctuations in the floating potential to be on the order of $0.1 \mathrm{~V}$ which is a fraction of the measured electron temperature. The EEDF was measured with a dynamic range up to $74 \mathrm{~dB}$. Typical Langmuir probe traces are shown in Figure 2 for several radial positions in the target chamber, $33 \mathrm{~cm}$ from the source-target interface. It is observed that the ion-saturation current is larger in the radial centre of the chamber and the electron temperature is hotter. A discussion of these observations is given below.

The EEDF was determined from the Langmuir probe I-V characteristic. Double differentiating the collected electron current gives an expression for the EEDF according to the

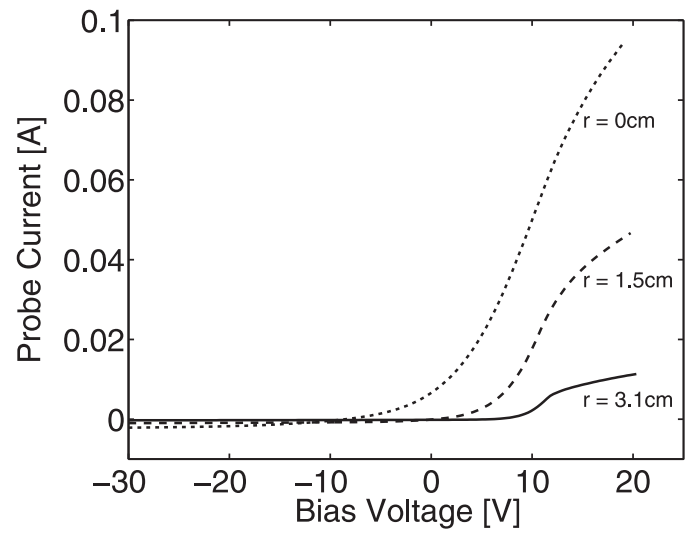

FIG. 2. Typical Langmuir probe I-V characteristics at a number of radial positions in a $200 \mathrm{~W}, 1.4$ mTorr plasma with a peak magnetic field of $600 \mathrm{G}$. Measurements were made $33 \mathrm{~cm}$ from the source-target interface.

Druyvesteyn method. ${ }^{17,24}$ In this work, the electron energy probability function (EEPF), $f_{p}(\epsilon)=\epsilon^{-1 / 2} F(\epsilon)$, is presented since on a logarithmic plot a Maxwellian distribution is a straight line and a Druyvesteyn distribution is a parabola.

In the presence of a magnetic field, the probe radius, $r_{p}$, and Debye length should be less than the Larmor radius, $r_{L}$, of the electrons so that the Druyvesteyn method is still valid and can be used without correction. ${ }^{17,25}$ The influence of the magnetic field on Langmuir probe results can be characterized by examining the ratio of the probe radius to the Larmor radius. ${ }^{26,27}$ For the experimental conditions described above, $r_{p} / r_{L} \ll 1$ throughout the majority of the reactor and the influence of the magnetic field on probe characteristics can be safely neglected. However, in the vicinity of the magnetic field peak, $r_{p} / r_{L}$ approaches 1 . In this regime, the EEDF is influenced by collisions and cross-field diffusion of electrons resulting from depletion near the probe tip. This is an effect more pronounced for low-energy electrons. As such, the magnetic field's influence manifests itself as a decrease in the electron saturation current. This depletes the EEDF at low energies and makes measurement of the plasma potential difficult. Density and temperature are calculated from the Langmuir probe's I-V characteristic by an Impedans analysis system. ${ }^{28}$ The ion saturation current and Laframboise theory are used to find the ion density. $T_{e}$ is calculated by finding the slope of the I-V characteristic between the floating potential and the plasma potential.

The influence of the magnetic pinch on the electron dynamics is demonstrated by measurement of the EEDF at a number of radial and axial positions (Figure 3 ). As can be seen from Figure 3(a), the EEPFs measured along the axis (at $r=0 \mathrm{~cm}$ ) of the reactor can be described as Maxwellian. The EEPF along this axis displays a large fraction of electrons with energy in the inelastic range $(\mathrm{E}>15 \mathrm{eV})$. It is observed that the EEPF along the axis maintains the same Maxwellian profile $43 \mathrm{~cm}$ into the diffusion chamber. At $r=1.5 \mathrm{~cm}$, the EEPF measured close to the source region (at $z=23 \mathrm{~cm}$ ), is similar to those measured on-axis, with a high fraction of electrons in the inelastic range. Further downstream at $z=33 \mathrm{~cm}$ and $z=43 \mathrm{~cm}$, the EEPFs are different. In particular, the EEPFs display a reduction of the high energy electrons above $15 \mathrm{eV}$, to a level below the dynamic 
(a)

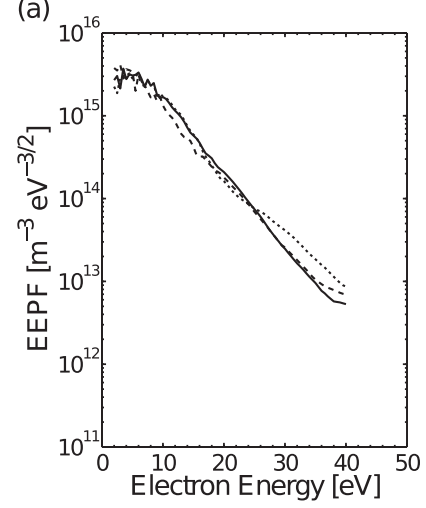

(b)

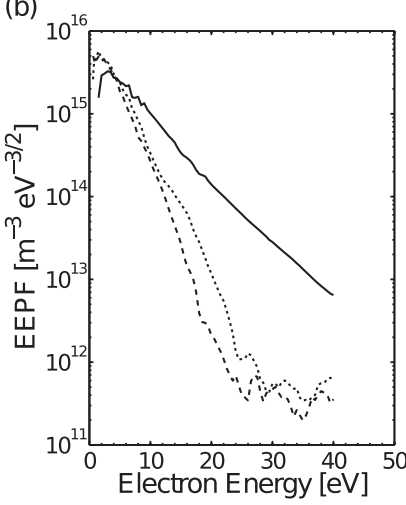

(c)

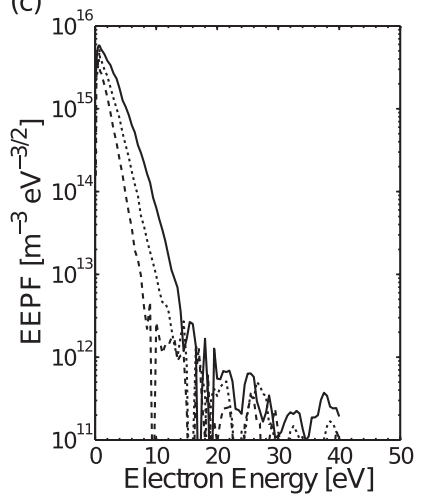

FIG. 3. Electron energy probability functions measured in a $200 \mathrm{~W}, 1.4$ mTorr plasma with a peak magnetic field of 600 G. Measurements are resolved both axially and radially with (a), (b), and (c) being measured at radial positions of $\mathrm{r}=0 \mathrm{~cm}, \quad \mathrm{r}=1.5 \mathrm{~cm}, \quad$ and $\mathrm{r}=3.1 \mathrm{~cm}$, respectively. measuring range of the Langmuir probe. The cooler electron temperature is indicated by the steeper slope compared with the axial centre EEPFs. However, the distribution still appears to be Maxwellian. At $r=3.1 \mathrm{~cm}$, cooler Maxwellian EEPFs are observed. In contrast to the on-axis measurements, the EEPFs at $r=3.1 \mathrm{~cm}$ display a decrease in both density and temperature with respect to the distance from the source region (increasing $z$ ). This is consistent with increased plasma confinement in the magnetic pinch region leading to a narrowing of the plasma column.

To further investigate plasma confinement in the downstream region, spatially resolved measurements were performed for ion density $\left(n_{i}\right)$, electron temperature $\left(T_{e}\right)$, and floating potential $\left(V_{f}\right)$. The axial scan was performed from $\mathrm{z}=23 \mathrm{~cm}$ to $\mathrm{z}=43 \mathrm{~cm}$ (with $\mathrm{z}=0$ being the source/diffusion chamber interface) at $2 \mathrm{~cm}$ intervals. The radial scan was across the plasma column at an intervals of $5^{\circ}$. The data are mapped onto an $r, z$ grid and are displayed in those dimensions in Figure 3. To give an indication of the density of data in these plots, there are a total of 121 measurements in the data set. The measurements of $n_{i}, T_{e}$, and $V_{f}$ show the existence of a plasma column that follows the converging field lines. The well-defined plasma column in the downstream region has a full width at half maximum (FWHM) between $3 \mathrm{~cm}$ and $4.5 \mathrm{~cm}$. The FWHM is observed to decrease with increasing $z$ indicative of increased plasma confinement (Figure 4(a)). It is observed that the axial ion density peaks in the vicinity of the magnetic pinch region $(z=42 \mathrm{~cm})$ with a density of $1.3 \times 10^{17} \mathrm{~m}^{-3}$. The increase in density in this region is small due to the low applied RF power and low magnetic field strength used for the measurements of Figure 4. At higher magnetic field strength, a decreased Larmor radius makes measurement of $T_{e}$ difficult. However, a qualitative comparison of high and low magnetic field operating conditions can be obtained by measuring the ion saturation current with an uncompensated Langmuir probe. The results reported in Figure 5 were acquired for an increased applied RF power of $500 \mathrm{~W}$ and operating pressure of $3 \mathrm{mTorr}$. Two magnetic field configurations were produced using a source solenoid current of $50 \mathrm{~A}$ and target solenoid currents of $304 \mathrm{~A}$ and $914 \mathrm{~A}$ to produce peak magnetic field strengths of $600 \mathrm{G}$ (dashed line) and $1800 \mathrm{G}$ (solid line), respectively. It can clearly be seen that the peak in ion-saturation current occurs close to the maximum magnetic field in the downstream region and increases with applied magnetic field.
While the electron density increases in the region of peak magnetic field, the electron temperature and floating potential remain roughly constant at $\sim 5 \mathrm{eV}$ and $\sim-8 \mathrm{~V}$, respectively. The effect of increased plasma confinement is most clearly seen in the two dimensional plot of the electron temperature (Figure 4(b)). Figure 4(b) shows that the electron temperature closely follows the magnetic field lines from $z=23 \mathrm{~cm}$ to $z=43 \mathrm{~cm}$. Along the magnetic field lines, the electron temperature is approximately constant, implying that electrons are able to equilibrate with one-another forming isotherms.

A negative floating potential (Figure 4(c)) is observed along the axis of the discharge and follows the magnetic field lines in a similar manner to the electron temperature. The floating potential displays an increasing negative radial gradient closer to the magnetic field pinch region. This radial variation of $T_{e}$ and $V_{f}$ indicates that the radial plasma potential is fairly flat. As discussed above, an applied magnetic
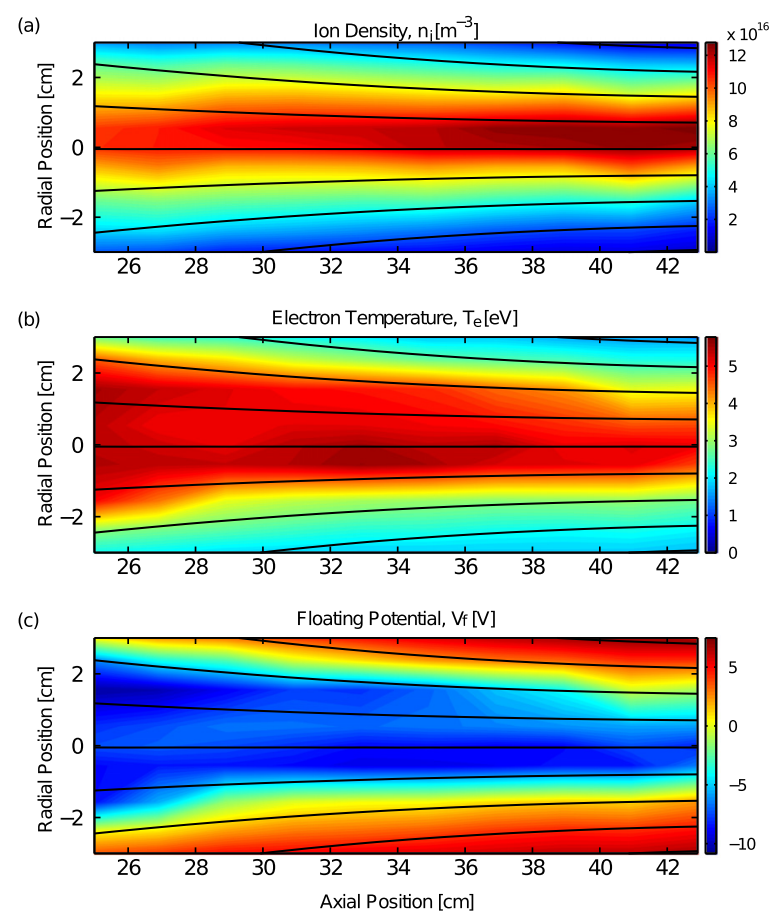

FIG. 4. Spatially resolved Langmuir probe results for a $200 \mathrm{~W}, 1.4$ mTorr argon plasma with a peak axial field of $600 \mathrm{G}$. Measurements have been overlaid with the magnetic field lines corresponding to the experimental setup described (solid black lines). 


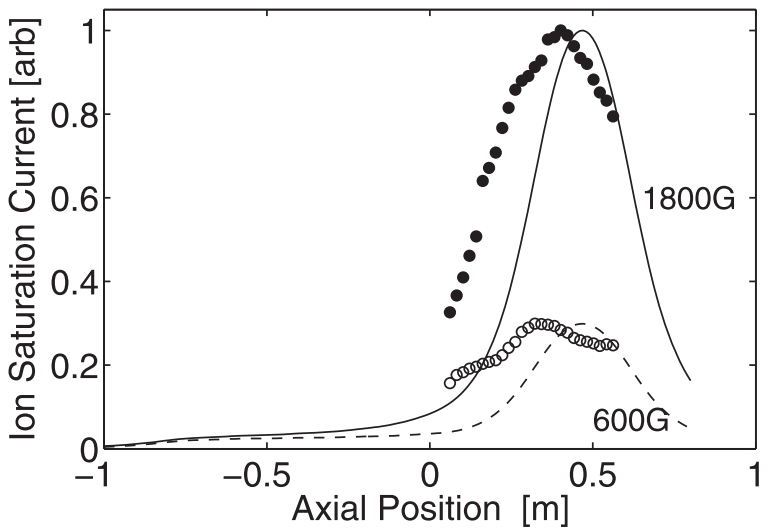

FIG. 5. Ion saturation current measured on-axis with an un-compensated Langmuir probe for a $500 \mathrm{~W}, 3$ mTorr plasma, and two magnetic field configurations. The on-axis magnetic field strength for these configurations has been normalised to the peak ion saturation value observed for that configuration (lines). Both sets of ion saturation data have been normalised to the maximum value in the $1800 \mathrm{G}$ case.

field in the plasma can deplete the electron saturation current making accurate measurement of the plasma potential difficult. Although not shown here, our direct measurement of the plasma potential using the Langmuir probe indicates a negative radial electric field, which would serve to increase ion confinement. This observation requires further investigation with alternative diagnostics such as a retarding field analyser. It has been suggested that a negative floating potential may be evidence for the presence of an energetic electron population. ${ }^{22,29}$ This is in agreement with the detection of a comparatively large proportion of high-energy electrons onaxis in the EEPFs. Inside the column, these electrons are transported efficiently from the source or are locally heated through a collisionless heating mechanism. Outside the column, the EEPFs demonstrate that the plasma is produced by cross-field diffusion of electrons, which is reduced as the magnetic field strength increases in the pinch region. Boswell and $\mathrm{Chen}^{9}$ attributed the increase in ion density with increasing magnetic field to higher plasma confinement, which leads to improved coupling of electrons to the helicon wave and hence increased ionization efficiency. We have previously shown, under similar operating conditions to that presented here, that the attenuation length for the helicon wave in our system is $35 \mathrm{~cm}$ and hence can reach the magnetic pinch region. Indeed, a recent model of wave fields in MAGPIE, using Maxwell's equations and a cold plasma dielectric tensor, show that the wave energy density is peaked in this downstream region. ${ }^{30}$ Modelling by Kinder and Kushner, ${ }^{31}$ using a two-dimensional hybrid plasma equipment model, showed that high energy electrons can be produced by the presence of electron trapping and wave heating via non-collisional acceleration of electrons in magnetically enhanced helicon sources. Our measurements support their work; however, further investigation is required to confirm the presence of non-collisional heating isolated within the plasma column.

In summary, spatially resolved profiles of plasma parameters and EEDFs have been measured in a low-pressure magnetically focused plasma. A well-confined plasma column is observed downstream of the source with an increase in density near the magnetic field maximum. EEDF measurements show that energetic electrons are efficiently transported along the axis of the reactor and form isotherms along magnetic field lines. Outside of the central plasma column, a low density and low temperature plasma is formed by cross-field diffusion. These results are important to understanding the underlying physics of such discharges in which power deposition may occur far from the antenna region. Future measurements will include spatially resolved ion energy distribution functions (IEDFs).

The authors would like to acknowledge support from the Australian Research Council through a Future Fellowship (FT100100825). The authors thank John Wach and Mark Gwynneth for technical support and Trevor LaFleur for helpful discussions.

${ }^{1}$ H. Amemiya, Jpn. J. Appl. Phys., Part 1 30, 2601 (1991).

${ }^{2}$ A. Aanesland, A. Meige, and P. Chabert, J. Phys.: Conf. Ser. 162, 012009 (2009).

${ }^{3}$ C. Charles, J. Phys. D: Appl. Phys. 42, 163001 (2009).

${ }^{4}$ G. A. Gary, Sol. Phys. 203, 71 (2001).

${ }^{5}$ U. Fantz, P. Franzen, W. Kraus, M. Berger, S. Christ-Koch, M. Fröschle, R. Gutser, B. Heinemann, C. Martens, P. McNeely, R. Riedl, E. Speth, and D. Wunderlich, Plasma Phys. Controlled Fusion 49, B563 (2007).

${ }^{6}$ R. Winglee, T. Ziemba, L. Giersch, J. Prager, J. Carscadden, and B. R. Roberson, Phys. Plasmas 14, 063501 (2007).

${ }^{7}$ R. Boswell, R. Porteous, A. Prytz, A. Bouchoule, and P. Ranson, Phys. Lett. A 91, 163 (1982).

${ }^{8}$ R. W. Boswell, Plasma Phys. Controlled Fusion 26, 1147 (1984).

${ }^{9}$ R. W. Boswell and F. F. Chen, IEEE Trans. Plasma Sci. 25, 1229 (1997).

${ }^{10}$ F. F. Chen, J. Vac. Sci. Technol. A 10, 1389 (1992).

${ }^{11}$ F. F. Chen, IEEE Trans. Plasma Sci. 25, 1245 (1997).

${ }^{12}$ X. M. Guo, J. Scharer, Y. Mouzouris, and L. Louis, Phys. Plasmas 6, 3400 (1999).

${ }^{13}$ Y. Mori, H. Nakashima, F. W Baity, R. H. Goulding, M. D. Carter, and D. O. Sparks, Plasma Sources Sci. Technol. 13, 424 (2004).

${ }^{14}$ X. Sun, C. Biloiu, R. Hardin, and E. E. Scime, Plasma Sources Sci. Technol. 13, 359 (2004).

${ }^{15}$ J. Prager, R. Winglee, T. Ziemba, B. Roberson, and G. Quetin, Plasma Sources Sci. Technol. 17, 025003 (2008).

${ }^{16}$ B. D. Blackwell, J. F. Caneses, C. M. Samuell, J. Wach, J. Howard, and C. Corr, Plasma Sources Sci. Technol. 21, 055033 (2012).

${ }^{17}$ V. A. Godyak and V. I. Demidov, J. Phys. D: Appl. Phys. 44, 233001 (2011).

${ }^{18}$ A. Aanesland, J. Bredin, P. Chabert, and V. Godyak, Appl. Phys. Lett. 100, 044102 (2012).

${ }^{19}$ K. Takahashi, C. Charles, R. Boswell, T. Kaneko, and R. Hatakeyama, Phys. Plasmas 14, 114503 (2007).

${ }^{20}$ K. Takahashi, C. Charles, R. Boswell, and R. Hatakeyama, Phys. Plasmas 15, 074505 (2008).

${ }^{21}$ K. Takahashi, C. Charles, R. Boswell, W. Cox, and R. Hatakeyama, Appl. Phys. Lett. 94, 191503 (2009).

${ }^{22}$ S. A. Cohen, X. Sun, N. M. Ferraro, E. E. Scime, M. Miah, S. Stange, N. S. Siefert, and R. F. Boivin, IEEE Trans. Plasma Sci. 34, 792 (2006).

${ }^{23}$ I. D. Sudit and F. F. Chen, Plasma Sources Sci. Technol. 3, 162 (1994).

${ }^{24}$ M. J. Druyvesteyn, Z. Phys. 64, 781 (1930).

${ }^{25}$ J. G. Laframboise and J. Rubinstein, Phys. Fluids 19, 1900 (1976).

${ }^{26}$ P. M. Chung, L. Talbot, and K. J. Touryan, Electric Probes in Stationary and Flowing Plasmas, Theory and Application (Springer-Verlag, Berlin, 1975).

${ }^{27}$ M. Tichy, P. Kudrna, J. F. Behnke, C. Csambal, and S. Klagge, J. Phys. IV France 7, 397 (1997)

${ }^{28}$ Impedans Ltd., ALP System ${ }^{T M}$ Langmuir Probe: Installation and User Guide. Revision 2.8 (Impedans Ltd., 2011).

${ }^{29}$ Y. Igarashi, K. Takahashi, and T. Fujiwara, IEEE Trans. Plasma Sci. 39, 2442 (2011).

${ }^{30}$ L. Chang, M. J. Hole, J. F. Caneses, G. Chen, B. D. Blackwell, and C. S. Corr, Phys. Plasmas 19, 083511 (2012).

${ }^{31}$ R. L. Kinder and M. J. Kushner, J. Appl. Phys. 90, 3699 (2001). 